\title{
Congenital Infection of Mice with Toxoplasma gondii Induces Minimal Change in Behavior and No Change in Neurotransmitter Concentrations
}

\author{
Author(s): David Goodwin, Terry C. Hrubec , Bradley G. Klein, Jeannine S. Strobl, Stephen R. Werre , \\ Qian Han , Anne M. Zajac , and David S. Lindsay \\ Source: Journal of Parasitology, 98(4):706-712. 2012. \\ Published By: American Society of Parasitologists \\ DOI: http://dx.doi.org/10.1645/GE-3068.1 \\ URL: http://www.bioone.org/doi/full/10.1645/GE-3068.1
}

BioOne (www.bioone.org) is a nonprofit, online aggregation of core research in the biological, ecological, and environmental sciences. BioOne provides a sustainable online platform for over 170 journals and books published by nonprofit societies, associations, museums, institutions, and presses.

Your use of this PDF, the BioOne Web site, and all posted and associated content indicates your acceptance of BioOne's Terms of Use, available at www.bioone.org/page/terms of use.

Usage of BioOne content is strictly limited to personal, educational, and non-commercial use. Commercial inquiries or rights and permissions requests should be directed to the individual publisher as copyright holder. 


\title{
CONGENITAL INFECTION OF MICE WITH TOXOPLASMA GONDII INDUCES MINIMAL CHANGE IN BEHAVIOR AND NO CHANGE IN NEUROTRANSMITTER CONCENTRATIONS
}

\author{
David Goodwin, Terry C. Hrubec ${ }^{\star}$ Bradley G. Klein, Jeannine S. Strobl†, Stephen R. Werre, Qian Hanł, Anne M. Zajac, and \\ David S. Lindsay§ \\ Department of Biomedical Sciences and Pathobiology, Virginia-Maryland Regional College of Veterinary Medicine, Virginia Tech, Blacksburg, \\ Virginia 24061-0342. e-mail: lindsayd@vt.edu
}

\begin{abstract}
We examined the effect of maternal Toxoplasma gondii infection on behavior and the neurotransmitter concentrations of congenitally infected CD-1 mice at 4 and $8 \mathrm{wk}$ of age when latent tissue cysts would be present in their brains. Because of sex-associated behavioral changes that develop during aging, infected female mice were compared with control females and infected male mice were compared with control males. Only the short memory behavior (distance between goal box and first hole investigated) of male mice congenitally infected with $T$. gondii was significantly different $(P<0.05)$ from that of uninfected control males at both 4 and 8 wk by using the Barnes maze test. The other parameters examined in the latter test, i.e., functional observational battery tests, virtual cliff, visual placement, and activity tests, were not significantly different $(P>0.05)$ at 4 and $8 \mathrm{wk}$. Concentrations of neurotransmitters and their metabolites (dopamine; 3,4-dihydroxyphenylacetic acid; homovanillic acid; norepinephrine; epinephrine; 3-methoxy-4hydroxyphenylglycol; serotonin; and 5-hydroxyindoleacetic acid) in the frontal cortex and striatum were not different $(P>0.05)$ between infected and control mice at $8 \mathrm{wk}$ of age. The exact mechanism for the observed effect on short-term memory in male mice is not known, and further investigation may help elucidate the molecular mechanisms associated with the proposed link between behavioral changes and $T$. gondii infection in animals. We were not able, however, to confirm the widely held belief that changes in neurotransmitters result from chronic $T$. gondii infection of the brain.
\end{abstract}

Toxoplasma gondii is an obligate intracellular protozoan parasite that infects most warm-blooded animals. The parasite has a heteroxenous life cycle, with felids serving as the only known definitive host. Rodents in particular have been studied extensively as intermediate hosts of $T$. gondii because they serve as a model for human infections and are commonly preyed upon by felids. Transmission of infection to the intermediate host can occur naturally in 1 of 3 ways, i.e., by ingestion of sporulated oocysts, by ingestion of tissue cysts, or by congenital transmission of tachyzoites. Transmission by the latter route may occur if a pregnant female acquires a primary $T$. gondii infection, allowing widespread bodily dissemination and infection of the fetus.

Congenital $T$. gondii infections are manifested in several ways. Clinical outcomes of infection can range from spontaneous abortions to only very mild symptoms in the offspring that are not apparent until later in life (see Jones et al., 2001). Severe developmental defects, including intra-cerebral calcification, hydrocephalus, and retinocorditis, may result from congenital toxoplasmosis. This wide range of symptoms results from several factors. The most important aspect is when fetal infection occurs during pregnancy. If the fetus is infected early in development, the outcome is more severe (Jones et al., 2001). Fetal infection early in pregnancy occurs at a lower frequency than infection later in pregnancy. Conversely, fetal infection later in pregnancy usually results in less severe disease and a more favorable outcome. Recent findings based on atypical $T$. gondii genotypes suggest, however, that human congenital toxoplasmosis may be more complex than previously appreciated (Lindsay and Dubey, 2011).

Many mental illnesses associated with cognitive impairments have no known etiology. It is believed that genetic and

Received 20 December 2012; revised 12 March 2012; accepted 2 April 2012.

* Via College of Osteopathic Medicine, Virginia Campus, Blacksburg, Virginia 24060.

$\dagger$ Department of Electrical and Computer Engineering, Virginia Tech, Blacksburg, Virginia 24061-0342.

\$Department of Biochemistry, Virginia Tech, Blacksburg, Virginia 240610342.

$\S$ To whom correspondence should be addressed.

DOI: 10.1645/GE-3068.1 environmental factors contribute to the development of several mental health disorders associated with cognitive problems. Suspected environmental factors include exposure to infectious agents such as $T$. gondii. Chronic postnatal $T$. gondii infections have been correlated with human behavioral changes (see Webster and McConkey, 2010). Rodents infected postnatally with $T$. gondii and tested after the infection has become chronic exhibit increased open field activity, decreased exploration, and loss of neophobia (Hutchinson et al., 1980; Hay et al., 1984; Webster et al., 1994; Vyas et al., 2007). People with mental health disorders associated with cognitive dysfunction have been shown to have a decreased intelligence quotient, decreased rate of learning, and difficultly interacting with society, and they may have memory impairment. The dopamine hypothesis, as a potential cause of cognitive deficits, proposes that hyperfunction of dopamine neurotransmission is involved in changes in prefrontal cortexassociated functions such as the rate of learning and memory. Consistent with the dopamine hypothesis, chronic toxoplasmosis has been reported to increase levels of dopamine in mice (Stibbs, 1985). Many of the behavioral changes in mice are similar to behavioral changes observed in humans living with cognitive impairments.

Stress experienced in utero (McClellan et al., 2006) also is currently being investigated as a potential environmental cause of cognitive impairments. Retrospective studies using archived maternal blood samples revealed that a significant number of offspring that developed schizophrenia, often associated with cognitive impairment, had been exposed to $T$. gondii infection in utero (Brown et al., 2005). It also has been suggested that maternal exposure to $T$. gondii infection is a risk factor for the development of psychoses other than schizophrenia (Brown et al., 2005; Mortensen et al., 2007; Xiao et al., 2009). Based on the aforementioned findings, we hypothesized that congenital $T$. gondii infection will result in exploratory, learning and memory, sensory, and motor behaviors that are significantly different between infected and uninfected mice and that dopamine levels also will be higher in infected mice. Because the onset of many mental illnesses is observed in late adolescence or early adulthood (Jablensky, 1997; McGorry et al., 2010), we examined 
congenitally $T$. gondii-infected mice pre- and post-sexual maturity to determine whether their behavior was different from uninfected age- and sex-matched controls. We also examined the effect of congenital toxoplasmosis on the concentration of dopamine, serotonin, and their catabolites and metabolites to determine whether significant changes occurred in the production or turnover of these neurotransmitters.

\section{MATERIALS AND METHODS}

\section{Congenital infections}

In total, 80 female and 40 male 5-wk-old CD-1 mice were used to produce the congenitally infected offspring used in this study. Food and water were given ad libitum for the duration of the experiment. The Institutional Animal Care and Use Committee at Virginia Tech approved the study. Because the experiment examined congenital transmission of $T$. gondii infections, surrogate females (dams) were used to eliminate negative effects of $T$. gondii infection on maternal parenting and lactation. Mice were acclimated to cages for 9 days. Eighty female mice were randomly allocated into 3 groups: $20 \mathrm{~T}$. gondii-infected pregnant females, 20 noninfected-pregnant females (negative controls), and 40 surrogates. The surrogate dams were used for both $T$. gondii-infected mice and noninfected negative control mice. Two females were co-housed with 1 male, and the females were examined every morning for the presences of a vaginal plug. Plug-positive females were removed, and this day was considered day 0 of pregnancy for the dams.

Experimental groups contained 20 control (not infected with $T$. gondii) and $20 \mathrm{~T}$. gondii-infected pregnant dams. Control mice received $500 \mu \mathrm{l}$ of Hanks' balanced salt solution (HBSS; Cellgro, Mediatech, Manassas, Virginia) orally on day 11 of pregnancy. Toxoplasma gondii-infected mice received 30 VEG tissue cysts (T. gondii genotype III) orally in $500 \mu \mathrm{l}$ of HBSS on day 11 of pregnancy. These cysts were collected from the brains of chronically infected CD-1 mice. All experimental dams (control and infected mice, but not surrogates) were killed after parturition, and their pups fostered to an age matched surrogate dam whose pups had been removed.

Pups were handled regularly pre- and post-weaning to acclimate them to handling. Pups were weaned at $3 \mathrm{wk}$ of age. This age was chosen because maternal immunoglobulin G disappears in the pups after 3 wk (Whitelaw and Urquhart, 1985). Mice were bled and ear notched for identification at $3.5 \mathrm{wk}$ of age. Their sera were examined for $T$. gondii antibodies at a 1:50 dilution by using the RH strain of $T$. gondii as antigen and immunofluorescent antibody (IFA) test procedures routinely used in our laboratory (Lindsay et al., 1990). This procedure established the infection status of pups and the percentage of transmission for pups born to infected females. Five seropositive mice of the same sex born to each infected mother, and 5 seronegative mice of the same sex born to each HBSS-treated mother were saved for behavioral testing. All behavioral assessments were made blind to treatment groups. Behaviors were examined at 4 and 8 wk because $T$. gondii tissue cysts are present in the brain at that time (Dubey, 1997).

At the end of the experiment, each mouse used for 8-wk Barnes maze testing was killed by cervical dislocation for brain dissections and neurotransmitter measurements. The remaining mice were killed by $\mathrm{CO}_{2}$ asphyxiation, and the brain from 1 mouse in each group was used for frontal cortex brain smears to determine the presence of $T$. gondii tissue cysts.

\section{Barnes maze apparatus and testing}

The Barnes maze is a spatial memory and learning test. The maze is used to examine short-term memory, acquisition of tasks, open field activity, and exploratory activity (Bach et al., 1995). The Barnes maze apparatus consisted of a circular Plexiglas platform $(90 \mathrm{~cm}$ in diameter) with 16 holes (each $5 \mathrm{~cm}$ in diameter) equally spaced around the perimeter, $5 \mathrm{~cm}$ from the edge, and $10.5 \mathrm{~cm}$ from each other (Fig. 1). The maze was mounted on a stand $91.5 \mathrm{~cm}$ from the floor. There were 2 locations, $180^{\circ}$ apart, for an escape box $(19 \times 9 \times 7.5 \mathrm{~cm}$ [length $\times$ width $\times$ height] $)$ to be mounted underneath a hole by a drawer mechanism. Visual cues were set up approximately $20 \mathrm{~cm}$ above and $45-50 \mathrm{~cm}$ from the horizontal edge of the circular maze. Cues were positioned in such a way as to not be identifiable with a specific hole. Video imaging equipment was set up approximately

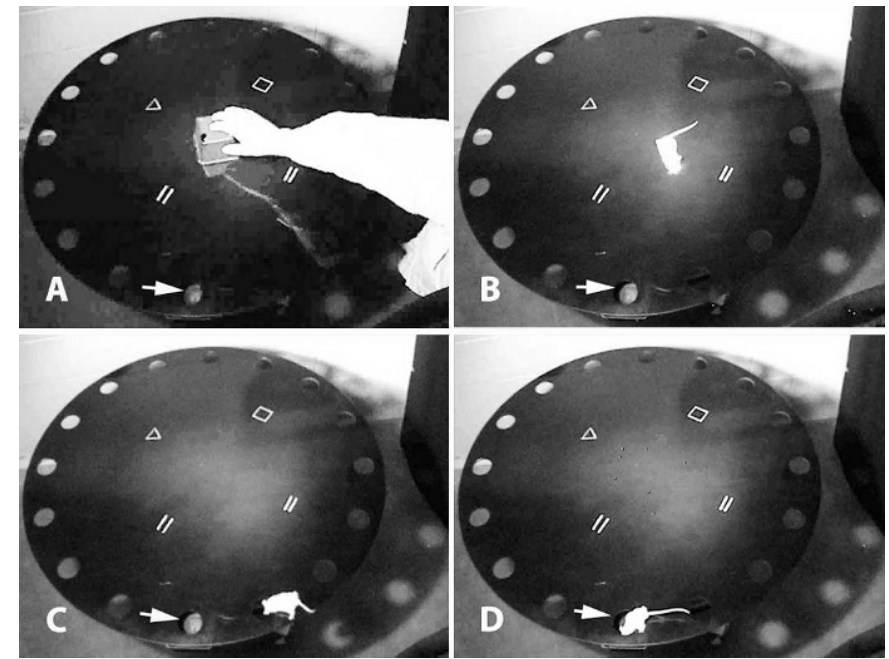

Figure 1. Barnes maze apparatus (arrow $=$ goal box) used to examine mouse behavior. (A) Beginning of the test with mouse in the start box in the center of the maze. (B) Mouse demonstrating inactive time. (C) Mouse investigating a wrong hole. (D) Mouse entering the goal box.

$149 \mathrm{~cm}$ above and $12 \mathrm{~cm}$ from the horizontal edge of the circular maze. The maze was set in the corner of a room, with a black curtain used for the other 2 walls. The corner of the room had white walls with black cues taped to the wall. The other 2 sides were black and had white cues attached to the curtain. The black curtain was positioned to block the experimenter from view. A 150-W light was suspended $106 \mathrm{~cm}$, almost directly above the center of the circular platform and a fan (Massey 8-inch high-velocity personal fan, Massey, Bentonville, Arkansas) was placed $68.5 \mathrm{~cm}$ above the maze pointed directly down at the center of the maze. The light and fan served as 2 forms of adverse stimuli. At the start of each trial, a mouse was placed in the center of the platform in a square start chamber $(8.8 \mathrm{~cm}$ in diameter and $8.1 \mathrm{~cm}$ in height).

Each mouse was randomly assigned, by a coin toss, to 1 of the 2 escape box locations; the escape box locations remained the same throughout the testing period. Testing was conducted daily for 7 days. On day 1 , each mouse was placed on the maze for $30 \mathrm{sec}$, without aversive stimuli and then physically placed in its designated goal box for $1 \mathrm{~min}$. On day 1 , the mice underwent training consisting of 2 test trials through the maze. On days $2-5$, the testing consisted of 2 consecutive trials followed by a third trial after $3-4-\mathrm{hr}$ rest. On the 6 th and 7 th days, each mouse was evaluated twice and once, respectively, totaling 17 trials per mouse.

For each trial, the mouse was placed in the center of the platform in a start chamber (Fig. 1A). Once placed on the platform, the fan and light were switched on and the mouse was kept in the start chamber for $10 \mathrm{sec}$. After $10 \mathrm{sec}$, the start chamber was lifted and the trial began. The trial ended once the mouse entered the goal box (forelimbs only, or both forelimbs and hind limbs), located the goal box (4 consecutive nasal investigations), or after a trial time of 5 min without entry or location of the goal box. When the mouse entered the goal box, the trial was stopped and the fan and light were turned off. If the mouse had located the goal box but did not enter, the trial was stopped, the mouse was then guided into the goal box, and the fan and light were turned off. If the mouse did not locate or enter the goal box after $5 \mathrm{~min}$, the trial was stopped, the mouse was placed into the goal box, and the fan and light were turned off. After $30 \mathrm{sec}$ in the goal box, the mouse was returned to its home cage. After a 3-5-min waiting period, the mouse was placed on the platform for the second daily trial. After the third trial, the mouse was returned to its home cage until the next day's session. The maze and goal box were cleaned with $70 \%$ ethanol after every trial.

Each trial was recorded digitally and then viewed at a later date for quantitative analysis. Video equipment consisted of a DCR-TRV530 digital 8 camcorder (Sony, Tokyo, Japan) connected via a Firewire cable to a Mac G (Apple Computer, Cupertino, California). Imovie (Apple Computer) was used to capture and save each trial. QuickTime ${ }^{\circledR}$ media player (Apple Computer) was used to view the video files. 
TABLE I. Behavioral parameters measured and results* of the Barnes maze test for 4-wk-old and 8-wk-old female and male congenitally Toxoplasma gondii-infected mice and non-infected controls.

\begin{tabular}{llll}
\hline \multicolumn{1}{c}{ Behavior } & \multicolumn{1}{c}{ Description } & Result 4 wk & Result 8 wk \\
\hline Inactive time & Initial period of inactivity in maze center when trial starts & Females only* & \multicolumn{1}{c}{ No difference } \\
Short memory & Distance between goal box and first hole investigated & Males only* & Males only* \\
Distance & Distance traveled to goal or reached by expiry of observation period & No difference & No difference \\
Errors & Visitation to a hole other than the goal box hole & No difference & No difference \\
Activity & No. of holes visited/active time (overall time minus inactive time) & No difference & No difference \\
Random search strategy & Passing through center of maze or skipping $>4$ holes & No difference & Females only* \\
Latency & Time elapsed from start to finish of trial & No difference & No difference \\
Task acquisition & Direct route to goal box with errors $\leq 2$ in 3 of 4 trials on 2 consecutive days & No difference & No difference \\
\hline
\end{tabular}

* Results were significantly different $(P \leq 0.05)$.

Barnes maze behavioral measures (Table I) were viewed and graded by 1 person (D.G.G.) who was blinded to the treatment status of the mice. The recorded videos were scored on inactive time, memory (distance to goal), distance, activity, random search strategy (center crossings), and latency.

Inactive time was the amount of time a mouse remained motionless in the center of the maze at the beginning of the trial. Inactive time is the amount of time required for a mouse to initially assess its environment. Short-term memory was assessed by the distance between the goal box and the first hole investigated. For example, if the first hole was adjacent to the goal box then the distance to goal value was recorded as 1 . Short-term memory is indicative of how well the mouse recognized its surrounding and remembered where the goal box was positioned (Bach et al., 1995). Distance was defined as how far the mouse traveled either before finding the goal box or the distance traveled during the 5-min observation time if the mouse was not successful in finding the goal box. Errors were recorded as the visitation to a hole other than the goal hole (Fig. 1C). Activity was calculated by the number of holes investigated divided by the active time (active time is overall time minus the inactive time) and defined as the amount of time spent investigating or exploring each hole. Random search strategy (center cross) (Fig. 1B) was defined as passing through the center of the maze, skipping more than 4 holes when traveling from hole to hole, or walking into the center of the maze and back out again. Random search strategy is a precursory approach where the mouse randomly searches for the goal box without a well-defined strategy. Acquiring the task was defined by a direct route to the goal box with errors $\leq 2$ in a trial. A mouse was considered to have acquired the task (learned the maze) if 3 of 4 trials on 2 consecutive days were successful at any point during the 7-day trial period (Fig. 1D). Latency was the time elapsed from trial start to finish.

The Barnes maze test was used to test 6 seronegative female mice, $8 \mathrm{~T}$. gondii-infected female mice, 7 seronegative male mice, and 7 T. gondiiinfected male mice at the 4 -wk time point. At the 8 -wk time point, 5 seronegative female mice, $7 \mathrm{~T}$. gondii-infected female mice, 6 seronegative male mice, and $7 T$. gondii-infected male mice were tested with the Barnes maze.

\section{Functional observation battery (FOB) tests}

FOB tests are designed to detect physiological changes, coordination, and startle and menace responses in mice (Moser et al., 1988; King et al., 2003). The specific physiologic parameters evaluated are listed in Table II. All of these parameters were graded on a "yes" or "no" scale Coordination was measured using 2 tests, i.e., the righting reaction test that tests the mouse's ability to right itself when placed on its back, and the rod test to see how well the mice hold onto a wooden rod. FOB tests also examined menace response by placing a pencil approximately $1-2 \mathrm{~cm}$ from the face of the mouse. If the mouse looked or turned away, then the mouse was scored with a "no", for no menace response. If the mouse acted aggressively to the pencil end, the mouse was scored as a "yes", i.e., the mouse acts menacingly to an object.

FOB tests used 9 seronegative female mouse litters, $4 T$. gondii-infected female mouse litters, 8 seronegative male mouse litters, and $5 T$. gondiiinfected male mouse litters for the 4-wk time point. At the 8-wk time point, 7 seronegative female mouse litters, $8 \mathrm{~T}$. gondii-infected female mouse litters, 7 seronegative male mouse litters, and $7 T$. gondii-infected male mouse litters were used for FOB testing.

\section{Visual placement}

Vision and coordination were evaluated by holding the mouse by its tail so that its head was $3.5-5 \mathrm{~cm}$ from the edge of the countertop and approximately $2.5 \mathrm{~cm}$ below the surface of the countertop. This stance encourages the mouse to reach up and out to the countertop to keep from dangling upside down (Fox, 1965). The mouse was then scored on a $3-$ point scale, i.e., (0) the mouse made no attempt to reach out, (1) the mouse attempted to reach out but could not grab the edge of the table, and (2) the mouse reached out and grabbed the edge of the table.

The visual placement test used 9 seronegative female mouse litters, $4 T$. gondii-infected female mouse litters, 8 seronegative mouse male litters, and $5 T$. gondii-infected male mouse litters for the 4-wk time point. At the 8 -wk time point, 7 seronegative female mouse litters, $8 T$. gondii-infected female mouse litters, 7 seronegative male mouse litters, and $7 T$. gondiiinfected male mouse litters were used for visual placement testing.

\section{Virtual cliff}

The virtual cliff (Adams et al., 2002) consisted of a box $(38 \times 38 \times$ $38 \mathrm{~cm}$ ) with a Plexiglas top surface that was half clear and half opaque. The virtual cliff was positioned $38 \mathrm{~cm}$ off the bottom of the box. Mice were placed in the center of the cliff with 1 front and hind limb of 1 side of the body on the clear portion and the contralateral limbs on the opaque portion. Mice were tested 3 times, and the initial position remained constant. Mice were scored depending on the side of the Plexiglas to which they traveled; a score of 1 was assigned for the clear side and a score of 2 for the opaque side.

The virtual cliff test used 9 seronegative female mouse litters, $4 \mathrm{~T}$. gondii-infected female mouse litters, 8 seronegative male mouse litters, and $5 T$. gondii-infected male mouse litters for the 4-wk time point. At the 8 -wk time point, 7 seronegative female mouse litters, $8 T$. gondii-infected female mouse litters, 7 seronegative male mouse litters, and $7 T$. gondiiinfected male mouse litters were used for virtual cliff testing.

\section{Rearing and open field activity}

Rearing tests look specifically at exploration of novel environments (Hutchinson et al., 1980). Four mice from each litter were individually placed in a box $(20 \times 42 \mathrm{~cm})$ for $5 \mathrm{~min}$. The number of times a mouse

TABLE II. Parameters evaluated in the functional observation battery (FOB) tests.*

\begin{tabular}{lll}
\hline Open field ataxia & Soft stool & Corneal bulging \\
Tremors/convulsions & Fecal stain & Partially closed eyes \\
Posture & Urine stain & Piloerection \\
Coat condition & Salivation & Dehydration \\
Tail condition & Nasal discharge & Protruding penis \\
Respiration & Oral discharge & Cool to touch cyanosis \\
Vocalization & Lacrimation & Weight gain or loss \\
Diarrhea & Ocular discharge & Activity \\
\hline
\end{tabular}

* None of these measured parameters was significantly different $(P<0.05)$. 
reared or lifted its forelimbs off the ground in an exploratory manner was counted. The box was cleaned with $70 \%$ ethanol between each mouse tested. The rearing test was not conducted at $4 \mathrm{wk}$. The rearing test used 6 seronegative female mouse litters, $9 T$. gondii-infected female mouse litters, 6 seronegative male mouse litters, and $7 T$. gondii-infected male mouse litters for the 8-wk time point.

Open field activity testing was carried out using a Photobeam Activity System (San Diego Instruments, San Diego, California) and a clear cage $(20 \times 42 \mathrm{~cm})$. The cage had a metal halo that surrounded the perimeter of the box, with 3 laser beams that were evenly spaced $10 \mathrm{~cm}$ apart, dividing the box into 4 evenly spaced sections $(20 \times 10 \mathrm{~cm})$. When the mouse moved from 1 quadrant to another quadrant, the laser beam was broken. A record of the beam breaks was recorded onto the computer. Mice were left in the open field for $5 \mathrm{~min}$, while beam breaks were recorded. The computer measured ambulations and beam breaks. The box was cleaned with $70 \%$ ethanol between each mouse tested. Ambulations were recorded as movement of a mouse through at least 3 of the 4 quadrants without back tracking. Beam breaks occurred when the mouse broke the beams in a non-successive order, indicating non-specific activity in the box.

The open field activity test was not conducted at $4 \mathrm{wk}$. The test used 7 seronegative female mouse litters, $7 T$. gondii-infected female mouse litters, 7 seronegative male mouse litters, and $7 T$. gondii-infected male mouse litters for the 8-wk observation time point.

\section{Neurotransmitter determinations}

Mice that completed the 8-wk Barnes maze test were used for neurotransmitter isolation and determination. The whole brain was removed and placed on ice and then cut in half along the sagittal plane. The striatum from both the right and the left hemispheres was removed and placed into a microfuge tube on ice. The frontal cortex from the right hemisphere was removed and placed in a microfuge tube on ice. The tissue was then weighed and 6 times the weight/volume of isoproterenol buffer, $\mathrm{pH} 4.7$, was added to the tissue for preservation of neurotransmitters. The brain tissue was then homogenized on ice followed by micro-centrifugation at $4 \mathrm{C}$ for $10 \mathrm{~min}$ at $15,000 \mathrm{~g}$. The supernatants were stored at $-80 \mathrm{C}$ until analysis by high-performance liquid chromatography (HPLC) was conducted. Striatal and cortical samples were analyzed for dopamine and its metabolites 3,4-dihydroxyphenylacetic acid and homovanillic acid. In addition, norepinephrine, epinephrine, and the metabolite 3-methoxy-4hydroxyphenylglycol and serotonin and its metabolite 5-hydroxyindoleacetic acid were analyzed.

Samples were examined in an 1100 Series HPLC (Agilent Technologies, Wilmington, Delaware) equipped with a degasser, a quaternary pump, and a refrigerated auto sampler (set at 4 C) connected to a 2465 electrochemical detector (Waters, Milford, Massachusetts). A C18 column (Pyramid EC $250 \times 4 \mathrm{~mm}$ i.d., $3 \mu \mathrm{m}$; Macherey Nagel Bethlehem, Pennsylvania) was used with a flow rate of $0.7 \mathrm{ml} / \mathrm{min}$. Column temperature was maintained at $35 \mathrm{C}$. The mobile phase composition was $5 \%$ methanol in aqueous sodium acetate $(50 \mathrm{mM})$, citric acid $(12.5 \mu \mathrm{M})$, EDTA $(134 \mu \mathrm{M})$, octane sulfonic acid $(230 \mu \mathrm{M})$, and sodium chloride $(2 \mathrm{mM}), \mathrm{pH} 4.7$. Isoproterenol $(1 \mu \mathrm{M})$ was used as the internal standard, and calibration curves of each neurotransmitter and metabolite were prepared using solutions of $10,50,100,250$, and $500 \mathrm{nM}$.

\section{Brain smears}

One mouse from each litter was examined for the presence of tissue cysts at the termination of the experiment. The cranial cavity of the mouse was opened, and approximately $0.1 \mathrm{~g}$ of the frontal cortex was removed, placed on a slide, crushed with a coverslip $\left(22 \times 22 \mathrm{~mm}^{2}\right)$, and examined at $\times 20$ using a BX60 microscope (Olympus, Tokyo, Japan) for the presence or absence of tissue cysts.

\section{Data analysis}

Transmission rates were compared between males and females by using logistic generalized estimating equations (GEEs; proc genmod). The linear model specified pup sero-status (positive vs. negative) as the outcome, sex as the predictor, logit as the link function, and binomial as the distribution. To request GEEs, dam IDs were specified as the clusters with an exchangeable working correlation matrix. $P$ values were based on type 3 Wald statistics. Sex ratios were compared between treatment groups by using the Wilcoxon rank sum test (proc npar1way).
Scatter plots for outcome against trial number showed that latency, active time, and distance and errors followed an exponential decay Subsequently, half-lives were generated for each of the 4 outcomes (for each mouse). The half-lives were then compared between the treatment groups using the Wilcoxon rank sum test. For outcomes that were slightly skewed (inactive time, distance to goal, activity, and center crosses), the treatment groups were compared using linear GEEs. The linear model specified behavioral measure as the outcome, treatment as a predictor, identity as the link function, and normal as the distribution. To request GEEs, mouse IDs were specified as clusters with an exchangeable working correlation matrix. $P$ values were based on the type 3 Wald statistics.

Only scores 2 and 1 were observed and recorded in the visual placement study. Accordingly, the treatment groups were compared using logistic GEEs (proc genmod). The linear model specified score as the outcome, treatment as a predictor, logit as the link function, and binomial as the distribution. To request GEEs, dam IDs within treatment were specified as the clusters with an exchangeable working correlation matrix. $P$ values were based on the type 3 Wald statistics.

The treatment groups were compared using logistic GEEs (proc genmod). The linear model specified FOB test as the outcome, treatment as a predictor, logit as the link function, and binomial as the distribution. To request GEEs, dam IDs within treatment were specified as the clusters with an exchangeable working correlation matrix. $P$ values were based on the type 3 Wald statistics.

In the virtual cliff experiment, the number of times a mouse went to the opaque side was used as the outcome for this section (score range, 0-3). After scoring, the treatment groups were compared using linear GEEs (proc genmod). The linear model specified score as the outcome, treatment as a predictor, identity as the link function, and normal as the distribution. To request GEEs, dam IDs within treatment were specified as clusters with an exchangeable working correlation matrix. $P$ values were based on the type 3 Wald statistics.

The effect of treatment on rearing was assessed using mixed model analysis of variance (ANOVA; proc mixed). The linear model included rearing as outcome, treatment as a fixed effect, and litter ID within treatment as the random effect. Ambulations and beam breaks were compared between treatments at each time point by using mixed model repeated measures ANOVA (proc glimmix). The linear model included ambulation or beam breaks as outcome, treatment as a fixed effect, and mouse within treatment as a random effect ( $\mathrm{G}$ side). For the $\mathrm{R}$ side of the model, an autoregressive 1 correlation matrix was specified. Denominator degrees of freedom were specified as KenwordRoger.

Neurotransmitter concentrations were compared between treatment groups using 1-way ANOVA (proc mixed). The linear model specified neurotransmitter as outcome, treatment as a fixed effect, and residual as the denominator degrees of freedom. The effect of treatment on epinephrine was assessed using Wilcoxon rank sum test.

Data were analyzed using SAS version 9.2 (SAS Institute, Cary, North Carolina). Because initial findings demonstrated sex-associated differences in mouse behavior between 4 and $8 \mathrm{wk}$ of age, mice were examined as 4 groups consisting of 4-wk-old female mice, 8-wk-old female mice, 4-wkold male mice, and 8-wk-old male mice. Statistical significance was set to a cutoff of $P<0.05$.

\section{RESULTS}

\section{Congenital transmission}

Fifteen of $20 T$. gondii-infected dams produced litters, resulting in 8 litters of $T$. gondi-infected females and 7 litters of $T$. gondiiinfected males. Twelve of 20 control dams produced litters, resulting in 6 litters of female and 7 litters of males. Congenital transmission of $T$. gondii was detected by IFA testing for 14 of 15 litters, by using our inoculation schedule. Of the 15 litters exposed to $T$. gondii in utero, there was a transmission success of $70 \%$ for males and $74 \%$ for females. No statistical differences $(P>0.05)$ in transmission success were detected between males and females. Of the 12 HBSStreated litters, none of the litters tested positive for $T$. gondii. The male-to-female pup ratio was 1.3 for $T$. gondii-infected litters and 1.0 for control litters, which is not significantly different $(P>0.05)$. 


\section{Behavior}

Barnes maze: The results of Barnes maze behavioral testing are summarized in Table I.

Behavior of congenitally infected female mice compared with seronegative control females in the Barnes maze: Behavior related to inactive time at $4 \mathrm{wk}$ was significantly different $(P<0.05)$ as was behavior related to random search strategy $(P<0.05)$ at $8 \mathrm{wk}$ for congenitally $T$. gondii-infected female mice (Table I). At $4 \mathrm{wk}$ of age, $T$. gondii-infected female mice spent $16.8 \mathrm{sec}$ sitting in the middle of the maze compared with $6.1 \mathrm{sec}$ for the female controls (a difference of $10.7 \mathrm{sec}$, with an SE of 5.260; $P<0.05$ ). At 8 wk of age, $T$. gondii-infected female mice had an average of 0.13 center crosses per trial, whereas control female mice had an average of 0.30 center crosses per trial (a difference of 0.17 , with an SE of $0.081 ; P<0.05)$. No other behavioral parameter measured in the Barnes maze test was significantly different $(P>$ 0.05 ) at either 4 or $8 \mathrm{wk}$ in congenitally $T$. gondii-infected female mice (Table I).

Behavior of congenitally infected male mice compared with seronegative control males in the Barnes maze: Behavior related to short-term memory at 4 and $8 \mathrm{wk}$ was significantly different $(P<$ 0.05 ) for congenitally $T$. gondii-infected male mice (Table I). At $4 \mathrm{wk}$, memory as measured by the first holes investigated by $T$. gondii-infected male mice were on average a distance of 3.1 holes from the goal box, whereas control mice first investigated a hole that was on average 4.1 holes from the goal box (a difference of 1 hole, with an SE of $0.476 ; P<0.05$ ). At 8 wk of age, the first hole investigated by infected male mice was 4.1 holes from the goal box compared with 3.0 holes for control male mice (a difference of 1.1 holes, with an SE of $0.389 ; P<0.05$ ). No other behavioral parameter measured in the Barnes maze test was significantly different $(P>0.05)$ at either 4 or $8 \mathrm{wk}$ in congenitally $T$. gondiiinfected male mice (Table I).

\section{Functional observation battery tests}

None of the measured parameters in the FOB tests were significantly different $(P>0.05)$ between groups (Table II).

\section{Virtual cliff and visual placement}

The direction of significant changes in virtual cliff parameters was different for males and females at $4 \mathrm{wk}$, but no significant differences were measured $8 \mathrm{wk}$. Congenitally infected female mice at 4 wk old went an average of 1.4 times to the opaque side compared with 1.8 times for control female mice (a difference of 0.4 , with an SE of $0.1 ; P<0.05$ ), whereas $T$. gondii-infected males went to the opaque side of the virtual cliff significantly more often $(P<0.05)$, an average of 1.9 times compared with 1.6 times in control male mice (a difference of 0.3, with an SE of 0.17 )

No significant differences $(P>0.05)$ between infected and control mice were found for male or female mice at either 4- or 8wk time points, when visual placement was assessed.

\section{Activity and rearing}

No significant differences $(P>0.05)$ were detected between any group of mice for activity (ambulations and beam breaks) or rearing.

\section{Neurotransmitter concentrations}

Neurotransmitters were only measured after the completion of the Barnes maze at the 8 -wk time point. There were no significant differences $(P>0.05)$ in neurotransmitter or metabolite concentrations for either $T$. gondii-infected females or males compared with controls (data not shown).

\section{Brain smears}

Twelve of the 15 mice representing each infected litter tested positive for $T$. gondii via IFA. Of these 12 positive mice, 11 tested positive for tissue cysts in the frontal cortex. None of the mice from control litters tested positive for $T$. gondii by IFA or brain smear.

\section{DISCUSSION}

\section{Congenital transmission rate of $T$. gondii}

Congenital $T$. gondii transmission rates varied during the course of pregnancy when exposure occurs (Ocampo and DuarteGandica, 2010). The highest rate of maternal-to-fetal transmission in mice occurs between days 10 and 12 of pregnancy. A transmission success of $73 \%$ in the present study is comparable to $90 \%$ reported by others (Wang et al., 2011). Equal transmission to male and female mice indicates both sexes were equally susceptible to congenital transmission of $T$. gondii.

\section{Behaviors}

Short-term memory, as measured by the distance of first holes investigated from the goal box, was the only behavior that was consistently significantly $(P<0.05)$ altered at 4 and 8 wk by congenital $T$. gondii infection in the present study, and this difference was observed only in male mice (Table I). However, the direction of this change reversed over time, making its significance difficult to interpret. Significant changes in inactive time behavior at $4 \mathrm{wk}$ and random search strategy at $8 \mathrm{wk}$ were observed in female mice, but their importance is debatable due to the lack of consistent findings at both 4 and $8 \mathrm{wk}$ for both measured parameters to determine a trend in behavior. Likewise, the parameters measured in the virtual cliff were conflicting at $4 \mathrm{wk}$ for male and female mice and not significantly different $(P>$ 0.05 ) at $8 \mathrm{wk}$, for male and female mice, resulting in no trend of behavior or change of behavior over time. This inconsistency of the behavior findings for virtual cliff testing brings their importance into question. No significant differences $(P>0.05)$ between infected and control mice were found for male or female mice at either 4- or 8-wk time points, when visual placement, activity, and rearing were assessed, indicating that these activities were not significantly affected by congenital $T$. gondii infection. This also suggests that our findings in the Barnes maze were not confounded by problems with motor behavior.

Our results indicate that congenital infection with $T$. gondii was capable of causing sex-dependent behavioral effects in parameters that have been associated with learning and memory, as well as visual perception that were not immediately observed. However, inconsistencies in either the persistence of changes over time, or in the direction of changes from 1 time point to another, make the importance of these changes difficult to evaluate at this time. These changes may represent subtle effects on memory and 
perception, if not other behaviors, in infected mice, that could become clearer if observations were extended to a time frame both earlier and later than that studied here.

\section{Toxoplasma gondii and neurotransmitters}

Little is known about chronic $T$. gondii infection of the brain and its influence on behavior as related to alterations in neurotransmitters. Neurotransmitter measurements for our mice demonstrated no statistical difference $(P>0.05)$ between congenitally $T$. gondii-infected mice and seronegative controls for both males and females in cortex or striatum. Our findings do not support the study of Stibbs (1985) who reported that dopamine was increased by $14 \%$ for chronically post-natally $T$. gondii-infected mice compared with seronegative controls. We found no differences $(P>0.05)$ in serotonin levels, in agreement with the findings of Stibbs (1985). Serotonin levels are unlikely to differ with course of infection. This is particularly relevant because the immune response to $T$. gondii infection induces interferon $\gamma$ that releases indoleamine 2,3-dioxygenase, degrading tryptophan (MacKenzie et al., 2007). This could decrease serotonin because tryptophan is a precursor to serotonin. The difference in findings for dopamine also may be attributable to us using a congenital model, whereas Stibbs (1985) used a post-natal chronic infection model for his 7-wk-old acutely and 12-wk-old chronically infected female mouse model. It is also possible Stibbs (1985) used a higher dose or a more virulent genotype of $T$. gondii for his infections, given that he did not report the dose or strain of $T$. gondii used for his study. This makes repetition of his experiments and interpretation of the results impossible. Results from Skallová et al. (2006) suggested that male mice may be more susceptible to subtle neurotransmitter changes than female mice; however, they did not examine neurotransmitter concentration in the brains of mice used in their study. Numerous other mouse behavioral studies cite Stibbs (1985) for his research demonstrating increased dopamine levels in the brains of mice chronically or acutely infected with $T$. gondii. However, Stibbs (1985) did not test behavior in his mice. The present study addressed these respective experimental deficiencies by examining both neurochemical and behavioral changes. The absence of neurochemical changes associated with our behavioral changes suggests that other transmitter systems than those measured may be involved, e.g., an amino acid or peptide, or mechanisms other than changes in transmitter content or turnover are responsible. Further complicating the alterations in neurotransmitter argument is that alteration in dopamine function can manifest in changes in dopamine receptor numbers and receptor sensitivity, both of which were not included in the scope of our experimental design.

\section{ACKNOWLEDGMENTS}

This work was supported by a grant from the Harvey W. Peters Research Foundation to T.C.H. and D.S.L. We thank Krystal Furman, Valarie Reinoso, Melanie French, Dustin Lucus, and Shannon Vires for assistance. We thank Drs. G. A. McConkey, A. Lucus, and S. M. Mitchell for useful comments on drafts of this paper.

\section{LITERATURE CITED}

Adams, B., T. Fitch, S. Chaney, and R. Gerlai. 2002. Altered performance characteristics in cognitive tasks: Comparisons of albino ICR and CD1 mouse strains. Behavioral Brain Research 133: 351361.
Bach, M. E., R. D. Hawkins, M. Osman, E. R. Kandel, and M. MAYFORD. 1995. Impairment of spatial but not contextual memory in CaMKII mutant mice with a selective loss of hippocampal LTP in the range of the theta frequency. Cell 81: 905-915.

Brown, A. S., C. A. Schaefer, C. P. Quesenberry, L. Liu, V. P. Babulas, AND E. S. SUSSER. 2005. Maternal exposure to toxoplasmosis and risk of schizophrenia in adult offspring. American Journal Psychiatry 162: 767-773.

Dubey, J. P. 1997. Tissue cyst tropism in Toxoplasma gondii: A comparison of tissue cyst formation in organs of cats and rodents fed oocysts. Parasitology 115: 15-20.

Fox, W. M. 1965. Reflex-ontogeny and behavioural development of the mouse. Animal Behavior 13: 234-241.

Hay, J., P. P. Aitken, And D. I. Graham. 1984. Toxoplasma infection and response to novelty in mice. Zeitschrift für Parasitenkunde 70: 575588.

Hutchinson, W. M., M. Bradley, W. M. Cheyne, B. W. Wells, and J. HAY. 1980. Behavioural abnormalities in Toxoplasma-infected mice. Annals of Tropical Medicine and Parasitology 74: 337-345.

JABLENSKY, A. 1997. The 100-year epidemiology of schizophrenia. Schizophrenia Research 28: 111-125.

Jones, J. L., A. Lopez, M. Wilson, J. Schulkin, And R. Gibbs. 2001. Congenital toxoplasmosis: A review. Obstetrical \& Gynecological Survey 56: 296-305.

King, M. D., D. S. Lindsay, S. Holladay, and M. Ehrich. 2003. Neurotoxicity and immunotoxicity assessment in CBA/J Mice with chronic Toxoplasma gondii infection and single-dose exposure to methylmercury. International Journal of Toxicology 22: 53-61.

Lindsay, D. S., B. L. Blagburn, and J. P. Dubey. 1990. Infection of mice with Neospora caninum (Protozoa: Apicomplexa) does not protect against challenge with Toxoplasma gondii. Infection and Immunity 58: $2699-2700$.

- AND J. P. Dubey. 2011. Toxoplasma gondii and the changing paradigm of congenital toxoplasmosis. Parasitology 138: 1829-1831.

MacKenzie, C. R., K. Heseler, A. Müller, and W. Däubener. 2007. Role of indoleamine 2,3-dioxygenase in antimicrobial defence and immuno-regulation: Tryptophan depletion versus production of toxic kynurenines. Current Drug Metabolism 8: 237-244.

McClellan, J. M., E. Susser, and M. C. King. 2006. Maternal famine, de novo mutations, and schizophrenia. Journal of the American Medical Association 296: 582-584.

McGorry, P. D., B. Nelson, S. Goldstone, and A. R. Yung. 2010. Clinical staging: A heuristic and practical strategy for new research and better health and social outcomes for psychotic and related mood disorders. Canadian Journal of Psychiatry 55: 486-497.

Mortensen, P. B., B. Norgand-Pedersen, B. L. Waltoft, T. L. Sorensen, D. HougaArd, and R. H. YolKen. 2007. Early infections of Toxoplasma gondii and the later development of schizophrenia. Schizophrenia Bulletin 33: 741-744.

Moser, V. C., J. P. McCormick, J. P. Creason, and R. C. MacPhail. 1988. Comparison of chlordimeform and carbaryl using a functional observational battery. Fundamental and Applied Toxicology 2: 189206

Ocampo, L. M., and I. Duarte-Gandica. 2010. A model of congenital toxoplasmosis transmission dynamics. Revista de Salud Pública 12: 317-326.

Regier, D. A., W. E. Narrow, D. S. Rae, R. W. Manderscheid, B. Z. Locke, AND F. K. Goodwin. 1993. The de facto US mental and addictive disorders service system. Epidemiologic catchment area prospective 1-year prevalence rates of disorders and services. Archives of General Psychiatry 50: 85-94.

Skallová, A., P. Kodym, D. Frynta, and J. Flegr. 2006. The role of dopamine in Toxoplasma-induced behavioural alterations in mice: An ethological and ethopharmacological study. Parasitology 133: 525535.

STibBs, H. H. 1985. Changes in brain concentrations of catecholamines and indoleamines in Toxoplasma gondii infected mice. Annals of Tropical Medicine and Parasitology 79: 153-157.

Vyas, A., S. K. Kim, N. Giacomini, J. C. Boothroyd, and R. M. SAPOLSKY. 2007. Behavioral changes induced by Toxoplasma infection of rodents are highly specific to aversion of cat odors. Proceedings of the National Academy of Sciences USA 104: 6442-6447. 
Wang, T., M. Liu, X. J. GaO, Z. J. ZhaO, X. G. Chen, and Z. R. Lun. 2011 Toxoplasma gondii: The effects of infection at different stages of pregnancy on the offspring of mice. Experimental Parasitology 127: 107-112

Webster, J. P., C. F. Brunton, and D. W. MacDonald. 1994. Effect of Toxoplasma gondii upon neophobic behaviour in wild brown rats, Rattus norvegicus. Parasitology 109: 37-43.

, AND G. A. McConKey. 2010. Toxoplasma gondii-altered host behaviour: Clues as to mechanism of action. Folia Parasitologica (Praha) 57: 95-104.
Whitelaw, D. D., and G. M. Urquhart. 1985. Maternally derived immunity in young mice to infection with Trypanosoma brucei and its potentiation by Berenil chemotherapy. Parasite Immunology 7: 289300

Xiao, J., S. L. Buka, T. D. Cannon, Y. Suzuki, R. P. Viscidi, E. F. TORREY, AND R. H. Yolken. 2009. Serological pattern consistent with infection with type I Toxoplasma gondii in mothers and risk of psychosis among adult offspring. Microbes and Infection 11: 10111018. 